\title{
Vibration-Powered Radiation of Quaking Magnetar
}

\author{
S. Bastrukov, ${ }^{1}$ J. W. Yu, ${ }^{1}$ R. X. Xu, ${ }^{1}$ and I. Molodtsova ${ }^{2}$ \\ ${ }^{1}$ State Key Laboratory of Nuclear Physics and Technology, School of Physics, Peking University, Beijing 100871, China \\ ${ }^{2}$ Laboratory of Informational Technologies, Joint Institute for Nuclear Research, Dubna 141980, Russia
}

Correspondence should be addressed to S. Bastrukov, bast.sergey@gmail.com

Received 5 November 2011; Accepted 20 December 2011

Academic Editor: D. Lazzati

Copyright () 2011 S. Bastrukov et al. This is an open access article distributed under the Creative Commons Attribution License, which permits unrestricted use, distribution, and reproduction in any medium, provided the original work is properly cited.

\begin{abstract}
In juxtaposition with the standard model of rotation-powered pulsar, the model of vibration-powered magnetar undergoing quake-induced torsional Alfvén vibrations in its own ultrastrong magnetic field experiencing decay is considered. The presented line of argument suggests that the gradual decrease of frequencies (lengthening of periods) of long-periodic-pulsed radiation detected from a set of X-ray sources can be attributed to magnetic-field-decay-induced energy conversion from seismic vibrations to magnetodipole radiation of quaking magnetar.
\end{abstract}

\section{Introduction}

There is a common recognition today that the standard (lighthouse) model of inclined rotator, lying at the base of current understanding of radio pulsars, faces serious difficulties in explaining the long-periodic $(2<P<12 \mathrm{~s})$-pulsed radiation of soft gamma repeaters (SGRs) and anomalous $\mathrm{X}$-ray pulsars (AXPs). The persistent X-ray luminosity of AXP/SGR sources $\left(10^{34}<L_{X}<10^{36} \mathrm{erg} \mathrm{s}^{-1}\right)$ is appreciably (10-100 times) larger than expected from a neutron star deriving radiation power from the energy of rotation with frequency of detected pulses. Such an understanding has come soon after the detection on March 5, 1979 of the first 0.2second long gamma burst [1], which followed by a 200second emission that showed a clear 8-second pulsation period [2], and association of this event to a supernova remnant known as N49 in the Large Magellanic Cloud [3]. This object is very young (only a few thousand years old), but the period of pulsating emission is typical of a much older neutron star. In works $[4,5]$ it has been proposed that discovered object, today designated SGR 0526-66, is a vibrating neutron star, that is, the detected for the first time long-periodic pulses owe their origin to the neutron star vibrations, rather than rotation as is the case with radio pulsars. During the following decades, the study of these objects has been guided by the idea $[6,7]$ that electromagnetic activity of magnetars, both AXP's and SGR's, is primarily determined by decay of ultrastrong magnetic field $\left(10^{14}<B<10^{16} \mathrm{G}\right)$ and that a highly intensive gamma bursts are manifestation of magnetar quakes [8-10].

In this paper we investigate in some detail the model of vibration-powered magnetar which is in line with the current treatment of quasiperiodic oscillations of outburst luminosity of soft gamma repeaters as being produced by Lorentz-force-driven torsional seismic vibrations triggered by quake. As an extension of this point of view, in this paper we focus on impact of the magnetic field decay on Alfvén vibrations and magnetodipole radiation generated by such vibrations. Before doing so, it seems appropriate to recall a seminal paper of Woltjer [11] who was first to observe that magnetic-flux-conserving core-collapse supernova can produce a neutron star with the above magnetic field intensity of typical magnetar. Based on this observation, Hoyle et al. [12] proposed that a strongly magnetized neutron star can generate magnetodipole radiation powered by energy of hydromagnetic, Alfvén, vibrations stored in the star after its birth in supernova event (see, also, [13]). Some peculiarities of this mechanism of vibration-powered radiation have been scrutinized in our recent work [14], devoted to radiative activity of pulsating magnetic white dwarfs, in which it was found that the necessary condition for the energy conversion from Alfvén vibrations into electromagnetic radiation is the decay of magnetic field. As was stressed, the magnetic field decay is one of the most conspicuous features distinguishing 
magnetars from normal rotation-powered pulsars. It seems not implausible, therefore, to expect that at least some of currently monitoring AXP/SGR-like sources are magnatars deriving power of pulsating magnetodipole radiation from energy of Alfvénic vibrations of highly conducting matter in the ultrastrong magnetic field experiencing decay.

In approaching Alfvén vibrations of neutron star in its own time-evolving magnetic field, we rely on the results of recent investigations [15-18] of both even parity poloidal and odd parity toroidal (according to Chandrasekhar terminology [19]) node-free Alfvén vibrations of magnetars in constant-in-time magnetic field. The extensive review of earlier investigations of standing-wave regime of Alfvénic stellar vibrations can be found in [20]. The spectral formula for discrete frequencies of both poloidal and toroidal $a$-modes in a neutron star with mass $M$, radius $R$, and magnetic field of typical magnetar, $B_{14}=B / 10^{14} \mathrm{G}$, reads

$$
\begin{gathered}
\omega_{\ell}=\omega_{A} s_{\ell}, \quad \omega_{A}=\frac{v_{A}}{R}=B \sqrt{\frac{R}{3 M}}, \quad M=\frac{4 \pi}{3} \rho R^{3}, \\
\nu_{A}=\frac{\omega_{A}}{2 \pi}=0.2055 B_{14} R_{6}^{1 / 2}, \quad\left(\frac{M}{M_{\odot}}\right)^{-1 / 2}, \mathrm{~Hz},
\end{gathered}
$$

where numerical factor $s_{\ell}$ is unique to each specific shape of magnetic field frozen in the neutron star of one and the same mass $M$ and radius $R$.

\section{Alfvén Vibrations of Magnetar in Time-Evolving Magnetic Field}

In above cited work it was shown that Lorentz-force-driven shear node-free vibrations of magnetar in its own magnetic field field $\mathbf{B}$ can be properly described in terms of material displacements $\mathbf{u}$ obeying equation of magneto-solid-mechanics

$$
\rho \ddot{\mathbf{u}}(\mathbf{r}, t)=\frac{1}{4 \pi}[\nabla \times[\nabla \times[\mathbf{u}(\mathbf{r}, t) \times \mathbf{B}(\mathbf{r}, t)]]] \times \mathbf{B}(\mathbf{r}, t),
$$

$$
\dot{\mathbf{u}}(\mathbf{r}, t)=[\boldsymbol{\omega}(\mathbf{r}, t) \times \mathbf{r}], \quad \boldsymbol{\omega}(\mathbf{r}, t)=A_{t}[\nabla \chi(r)] \dot{\alpha}(t) .
$$

The field $\dot{\mathbf{u}}(\mathbf{r}, t)$ is identical to that for torsion node-free vibrations restored by Hooke's force of elastic stresses [18, 21] with $\chi(\mathbf{r})=A_{\ell} f_{\ell}(r) P_{\ell}(\cos \theta)$, where $f_{\ell}(r)$ is the nodeless function of distance from the star center and $P_{\ell}(\cos \theta)$ is Legendre polynomial of degree $\ell$ specifying the overtone of toroidal mode. In (4), the amplitude $\alpha(t)$ is the basic dynamical variable describing time evolution of vibrations, which is different for each specific overtone; in what follows, we confine our analysis to solely one quadrupole overtone. The central to the subject of our study is the following representation of the time-evolving internal magnetic field:

$$
\mathbf{B}(\mathbf{r}, t)=B(t) \mathbf{b}(\mathbf{r})
$$

where $B(t)$ is the time-dependent intensity and $\mathbf{b}(\mathbf{r})$ is dimensionless vector-function of the field distribution over the star volume. Scalar product of (1) with the separable form of material displacements

$$
\mathbf{u}(\mathbf{r}, t)=\mathbf{a}(\mathbf{r}) \alpha(t)
$$

followed by integration over the star volume leads to equation for amplitude $\alpha(t)$ having the form of equation of oscillator with time-dependent spring constant

$$
\mathcal{M} \ddot{\alpha}(t)+\mathcal{K}(t) \alpha(t)=0 .
$$

The total vibration energy and frequency are given by

$$
\begin{gathered}
E_{A}(t)=\frac{\mathcal{M} \dot{\alpha}^{2}(t)}{2}+\frac{\mathcal{K}(B(t)) \alpha^{2}(t)}{2}, \\
\omega(t)=\sqrt{\frac{\mathcal{K}(t)}{\mathcal{M}}}=B(t) \kappa, \quad \kappa=\sqrt{\frac{R}{3 M}} s .
\end{gathered}
$$

It follows that

$$
\frac{d E_{A}(t)}{d t}=\frac{\alpha^{2}(t)}{2} \frac{d \mathcal{K}(B)}{d B} \frac{d B(t)}{d t} .
$$

This shows that the variation in time of magnetic field intensity in quaking magnetar causes variation in the vibration energy. In Section 3, we focus on conversion of energy of Lorentz-force-driven seismic vibrations of magnetar into the energy of magnetodipole radiation.

\section{Vibration-Powered Radiation of Quaking Magnetar}

The point of departure in the study of vibration energypowered magnetodipole emission of the star (whose radiation power, $\mathcal{P}$, is given by Larmor's formula) is

$$
\frac{d E_{A}(t)}{d t}=-\mathcal{P}(t), \quad \mathcal{P}(t)=\frac{2}{3 c^{3}} \delta \ddot{\mu}^{2}(t) .
$$

We consider a model of a quaking neutron star whose torsional magnetomechanical oscillations are accompanied by fluctuations of total magnetic moment preserving its initial (in seismically quiescent state) direction: $\boldsymbol{\mu}=\boldsymbol{\mu} \mathbf{n}=$ constant. The total magnetic dipole moment should execute oscillations with frequency $\omega(t)$ equal to that for magnetomechanical vibrations of stellar matter, which are described by equation for $\alpha(t)$. This means that $\delta \boldsymbol{\mu}(t)$ and $\alpha(t)$ must obey equations of similar form, namely,

$$
\begin{gathered}
\delta \ddot{\boldsymbol{\mu}}(t)+\omega^{2}(t) \delta \boldsymbol{\mu}(t)=0, \\
\ddot{\alpha}(t)+\omega^{2}(t) \alpha(t)=0, \quad \omega^{2}(t)=B^{2}(t) \kappa^{2} .
\end{gathered}
$$

It is easy to see that (11) can be reconciled if

$$
\delta \boldsymbol{\mu}(t)=\boldsymbol{\mu} \alpha(t) .
$$

Given this, we arrive at the following law of magnetic field decay:

$$
\begin{array}{r}
\frac{d B(t)}{d t}=-\gamma B^{3}(t), \quad \gamma=\frac{2 \mu^{2} \kappa^{2}}{3 \mathcal{M} c^{3}}=\text { constant, } \\
B(t)=\frac{B(0)}{\sqrt{1+t / \tau}}, \quad \tau^{-1}=2 \gamma B^{2}(0) .
\end{array}
$$


The last equation shows that the lifetime of quake-induced vibrations in question substantially depends upon the intensity of initial (before quake) magnetic field $B(0)$ : the larger the $B(0)$ the shorter the $\tau$. For neutron stars with one and the same mass $M=1.4 M_{\odot}$ and radius $R=15 \mathrm{~km}$, and magnetic field of typical pulsar $B(0)=10^{12} \mathrm{G}$, we obtain $\tau \sim 3 \times 10^{7}$ years, whereas for magnetar with $B(0)=10^{14} \mathrm{G}, \tau \sim 7 \times 10^{3}$ years.

The equation for vibration amplitude

$$
\begin{gathered}
\ddot{\alpha}(t)+\omega^{2}(t) \alpha(t)=0, \\
\omega^{2}(t)=\frac{\omega^{2}(0)}{1+t / \tau}, \quad \omega(0)=\omega_{A} \kappa .
\end{gathered}
$$

with help of substitution $s=1+t / \tau$ is transformed to

$$
s \alpha^{\prime \prime}(s)+\beta^{2} \alpha(s)=0, \quad \beta^{2}=\omega^{2}(0) \tau^{2}=\text { constant }
$$

permitting general solution [22]. The solution of this equation, obeying two conditions $\alpha(t=0)=\alpha_{0}$ and $\alpha(t=\tau)=0$, can be represented in the form

$$
\begin{gathered}
\alpha(t)=C s^{1 / 2}\left\{J_{1}(z(t))-\eta Y_{1}(z(t))\right\}, \quad z(t)=2 \beta s^{1 / 2}(t), \\
\eta=\frac{J_{1}(z(\tau))}{Y_{1}(z(\tau))}, \quad C=\alpha_{0}\left[J_{1}(z(0))-\eta Y_{1}(z(0))\right]^{-1},
\end{gathered}
$$

where $J_{1}(z)$ and $Y_{1}(z)$ are Bessel functions [23] and

$$
\alpha_{0}^{2}=\frac{2 \bar{E}_{A}(0)}{M \omega^{2}(0)}=\frac{2 \bar{E}_{A}(0)}{K(0)}, \quad \omega^{2}(0)=\frac{K(0)}{M} .
$$

Here by $\bar{E}_{A}(0)$, the average energy stored in torsional Alfvén vibrations of magnetar is understood. If all the detected energy $E_{\text {burst }}$ of X-ray outburst goes in the quake-induced vibrations, $E_{\text {burst }}=E_{A}$, then the initial amplitude $\alpha_{0}$ is determined unambiguously. The impact of magnetic field decay on frequency and amplitude of torsional Alfvén vibrations in quadrupole overtone is illustrated in Figure 1, where we plot $\alpha(t)$ with pointed out parameters $\beta$ and $\eta$. The magneticfield-decay-induced lengthening of period of pulsating radiation (equal to period of vibrations) is described by

$$
\begin{gathered}
P(t)=P(0)\left[1+\left(\frac{t}{\tau}\right)\right]^{1 / 2}, \quad \dot{P}(t)=\frac{1}{2 \tau} \frac{P(0)}{[1+(t / \tau)]^{1 / 2}}, \\
\tau=\frac{P^{2}(0)}{2 P(t) \dot{P}(t)}, \quad P(0)=\frac{2 \pi}{\kappa B(0)} .
\end{gathered}
$$

On comparing $\tau$ given by (14) and (19), one finds that interrelation between the equilibrium value of the total magnetic moment $\mu$ of a neutron star of mass $M=1.4 M_{\odot}$ and radius $R=10 \mathrm{~km}$ vibrating in quadrupole overtone of toroidal $a$-mode is given by

$$
\begin{gathered}
\mu=A(M, R) \sqrt{P(t) \dot{P}(t)}, \quad A=\sqrt{\frac{3 \mathcal{M} c^{3}}{8 \pi^{2}}}, \\
\mu=3.8 \times 10^{37} \sqrt{P(t) \dot{P}(t)} \mathrm{G} \mathrm{cm}^{3} .
\end{gathered}
$$
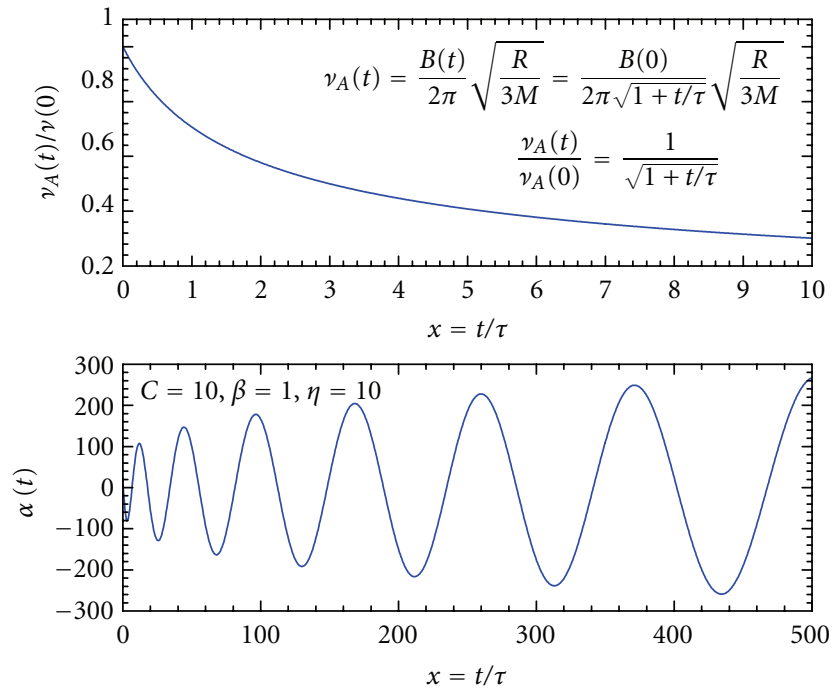

FIgure 1: (Color online) The figure illustrates the effect of magnetic field decay on the vibration frequency and amplitude of quadrupole toroidal $a$-mode presented as functions of $x=t / \tau$.

For a sake of comparison, in the considered model of vibration-powered radiation, the equation of magnetic field evolution is obtained in a similar fashion as that for the angular velocity $\Omega(t)$ in the standard model of rotation-powered neutron star which rests on

$$
\begin{gathered}
\frac{d E_{R}}{d t}=-\frac{2}{3 c^{3}} \delta \ddot{\mu}^{2}(t), \\
E_{R}(t)=\frac{1}{2} I \Omega^{2}(t), \quad I=\frac{2}{5} M R^{2}, \\
\delta \ddot{\boldsymbol{\mu}}(t)=[\boldsymbol{\Omega}(t) \times[\boldsymbol{\Omega}(t) \times \boldsymbol{\mu}]], \\
\boldsymbol{\mu}=\mu \mathbf{n}, \quad \mu=\frac{1}{2} B R^{3}
\end{gathered}
$$

which lead to

$$
\begin{gathered}
\dot{\Omega}(t)=-K \Omega^{3}(t), \quad K=\frac{2 \mu_{\perp}^{2}}{3 I c^{3}}, \quad \mu_{\perp}=\mu \sin \theta, \\
\Omega(t)=\frac{\Omega(0)}{\sqrt{1+t / \tau}}, \quad \tau^{-1}=2 K \Omega^{2}(0),
\end{gathered}
$$

where $\theta$ is angle of inclination of $\boldsymbol{\mu}$ to $\boldsymbol{\Omega}(t)$. The time evolution of $P(t), \dot{P}(t)$ and expression for $\tau$ are too described by (19). It is these equations which lead to widely used exact analytic estimate of magnetic field on the neutron star pole: $B=\left[3 I c^{3} /\left(2 \pi^{2} R^{6}\right)\right]^{1 / 2} \sqrt{P(t) \dot{P}(t)}$. For a neutron star of mass $M=M_{\odot}$ and radius $R=13 \mathrm{~km}$, one has $B=$ $3.210^{19} \sqrt{P(t) \dot{P}(t)}$ G. Thus, the substantial physical difference between vibration- and rotation-powered neutron star models is that in the former model the elongation of pulse period is attributed to magnetic field decay, whereas in the latter one the period lengthening is ascribed to the slow-down of rotation $[24,25]$. 


\section{Summary}

The last two decades have seen a growing understanding that magnetic field decay is the key property distinguishing magnetars from pulsars. The magnetic fields frozen in the immobile matter of pulsars (most of which are fairly stable to starquakes) operate like a passive (unaltered in time) promoter of their persistent radiation along the axis of dipole magnetic moment of the star inclined to the rotation axis. The presented treatment of spindown effect emphasizes kinematic nature of time evolution of pulsar magnetic moment as brought about by rapid rotation with gradually decreasing frequency and showing that rotation does not affect intensity of its internal magnetic field. Unlike pulsars, magnetars (SGR-like sources) are isolated quaking neutron stars whose seismic and radiative activity is thought to be dominated by decay of magnetic field. Perhaps the most striking manifest of seismic vibrations of magnetar are quasiperiodic oscillations of outburst luminosity rapidly decreasing (in several hundred seconds) from about $L_{X} \sim 10^{44} \mathrm{erg} \mathrm{s}^{-1}$ in giant flare to about $L_{X} \sim 10^{34} \mathrm{erg} \mathrm{s}^{-1}$ in quiescent regime of long-periodic X-ray emission. As was emphasized, this lowest value of luminosity is well above of the rate of energy of rotation with period equal to that of detected emission. With all above in mind, we have set up a model of vibration-powered radiation of quaking magnetar in which the key role is attributed to magnetic field decay. The most striking outcome of presented line of argument is the gradual decrease of frequencies (lengthening of periods) of magnetodipole radiation (pulsating with the frequency of toroidal $a$-mode of seismic vibrations) owing its origin to magnetic field decay. Remarkably, this prediction of the model is consistent with data on gradual decrease of frequency of pulsed X-ray emission of such magnetars as 1E 2259.1+586 [26] and XTE J1810-197 [27]. It is hoped, therefore, that the theoretical results presented here can be efficiently utilized as a guide in observational quest for vibration-powered neutron stars.

\section{Acknowledgments}

This work is supported by the National Natural Science Foundation of China (Grant nos. 10935001 and 10973002), the National Basic Research Program of China (Grant no. 2009CB824800), and the John Templeton Foundation.

\section{References}

[1] E. P. Mazets, S. V. Golenetskii, V. N. Il'inskii, R. L. Aptekar', and Y. A. Guryan, "Observations of a flaring X-ray pulsar in Dorado,” Nature, vol. 282, no. 5739, pp. 587-589, 1979.

[2] S. Barat, G. Chambon, K. Hurley et al., "Evidence for periodicity in a $\gamma$-ray burst," Astronomy and Astrophysics, vol. 79, no. 3, pp. L24-L25, 1979.

[3] T. L. Cline, "Detection of a fast, intense and unusual $\gamma$-ray transient," Astrophysical Journal, vol. 237, pp. L1-L5, 1980.

[4] R. Ramaty, S. Bonazzola, T. L. Cline, D. Kazanas, P. Mészáros, and R. E. Lingenfelter, "Origin of the 5 March $1979 \gamma$-ray transient: a vibrating neutron star," Nature, vol. 287, no. 5778, pp. 122-124, 1980.
[5] R. Ramaty, "Vibrating neutron star," Sky and Telescope, vol. 60, p. $484,1980$.

[6] R. C. Duncan and C. Thompson, "Formation of very strongly magnetized neutron stars: implications for gamma-ray bursts," Astrophysical Journal, vol. 392, no. 1, pp. L9-L13, 1992.

[7] B. Paczyński, "Gb 790305 as a very strongly magnetized neutron star," Acta Astronomica, vol. 42, no. 3, pp. 145-153, 1996.

[8] O. Blaes, R. Blandford, P. Goldreich, and P. Madau, "Neutron starquake models for $\gamma$-ray bursts," Astrophysical Journal, vol. 343, pp. 839-848, 1989.

[9] B. Cheng, R. I. Epstein, R. A. Guyer, and A. C. Young, "Earthquake-like behaviour of soft $\gamma$-ray repeaters," Nature, vol. 382, no. 6591, pp. 518-520, 1996.

[10] K. Y. Ding and K. S. Cheng, "Oscillation-induced $\gamma$-ray emission from dead pulsars: a model for the delayed $\mathrm{GeV}$ emission in gamma-ray bursts," Monthly Notices of the Royal Astronomical Society, vol. 287, no. 3, pp. 671-680, 1997.

[11] L. Woltjer, "X-rays and type i supernova remnants," Astrophysical Journal, vol. 140, pp. 1309-1313, 1964.

[12] F. Hoyle, J. V. Narlikar, and J. A. Wheeler, "Electromagnetic waves from very dense stars," Nature, vol. 203, no. 4948, pp. 914-916, 1964.

[13] F. Pacini, "The early history of neutron stars," in Proceedings of the MEASRIM No1, A. Hady and M. I. Wanas, Eds., p. 75, 2008.

[14] S. I. Bastrukov, J. W. Yu, R. X. Xu, and I. V. Molodtsova, "Radiative activity of magnetic white dwarf undergoing Lorentz-force-driven torsional vibrations," Modern Physics Letters A, vol. 26, no. 5, pp. 359-366, 2011.

[15] S. Bastrukov, J. Yang, M. Kim, and D. Podgainy, "Magnetic properties of neutron star matter and pulsed gamma emission of soft gamma repeaters," in Current High-Energy Emission Around Black Holes, H. Lee and H.-Y. Chang, Eds., pp. 334342, World Scientific, Singapore, 2002.

[16] S. I. Bastrukov, G. T. Chen, H. K. Chang, I. V. Molodtsova, and D. V. Podgainy, "Torsional nodeless vibrations of a quaking neutron star restored by the combined forces of shear elastic and magnetic field stresses," Astrophysical Journal, vol. 690, no. 1, pp. 998-1005, 2009.

[17] S. I. Bastrukov, H. K. Chang, I. V. Molodtsova, E. H. $\mathrm{Wu}, \mathrm{G}$. T. Chen, and S. H. Lan, "Frequency spectrum of toroidal Alfvén mode in a neutron star with Ferraro's form of nonhomogeneous poloidal magnetic field," Astrophysics and Space Science, vol. 323, no. 3, pp. 235-242, 2009.

[18] S. Bastrukov, I. Molodtsova, J. Takata, H. K. Chang, and R. $\mathrm{X}$. Xu, "Alfvén seismic vibrations of crustal solid-state plasma in quaking paramagnetic neutron star," Physics of Plasmas, vol. 17, no. 11, Article ID 112114, 10 pages, 2010.

[19] S. Chandrasekhar, "Hydromagnetic oscillations of a fluid sphere with internal motions," Astrophysical Journal, vol. 124, p. 571, 1965.

[20] P. Ledoux and T. Walraven, "Variable stars," in Handbuch der Physik, S. Flügge, Ed., vol. 51, pp. 353-604, Springer, New York, NY, USA, 1958.

[21] S. I. Bastrukov, H. K. Chang, J. Takata, G. T. Chen, and I. V. Molodtsova, "Torsional shear oscillations in the neutron star crust driven by the restoring force of elastic stresses," Monthly Notices of the Royal Astronomical Society, vol. 382, no. 2, pp. 849-859, 2007.

[22] A. D. Polyanin and V. F. Zaitsev, Handbook of Nonlinear Partial Differential Equations, Chapman \& Hall, Boca Raton, Fla, USA, 2004.

[23] M. Abramowitz and I. Stegun, Handbook of Mathematical Functions, Dover, New York, NY, USA, 1972. 
[24] R. N. Manchester and J. H. Taylor, Pulsars, Freeman, San Francisco, Calif, USA, 1977.

[25] D. R. Lorimer and M. Kramer, Handbook of Pulsar Astronomy, Cambridge University Press, Cambridge, UK, 2004.

[26] F. P. Gavriil, V. M. Kaspi, and P. M. Woods, "Anomalous Xray pulsars: long-term monitoring and soft-gamma repeater like X-ray bursts," Advances in Space Research, vol. 33, no. 4, pp. 654-662, 2004.

[27] A. I. Ibrahim, C. B. Markwardt, J. H. Swank et al., "Discovery of a transient magnetar: XTE J1810-197," Astrophysical Journal, vol. 609, no. 1, pp. L21-L24, 2004. 

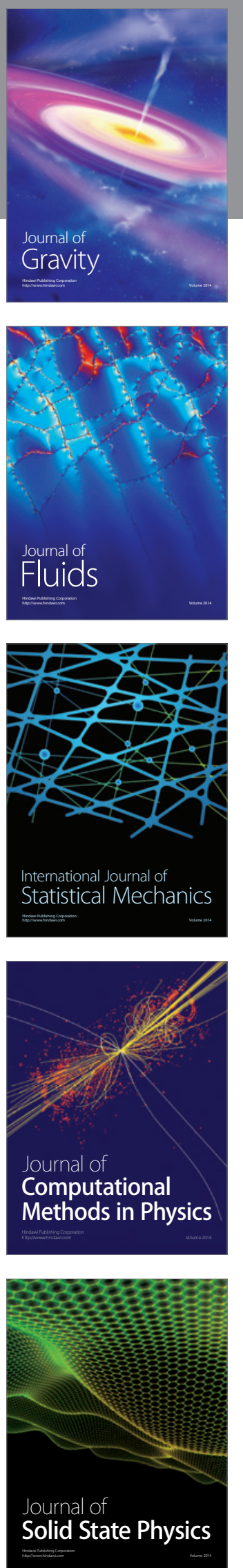

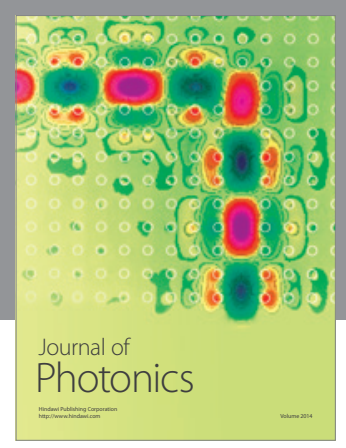

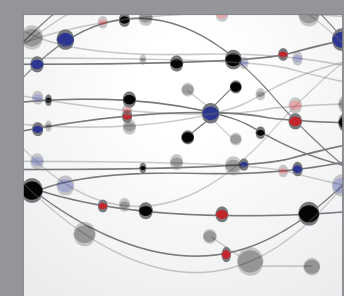

The Scientific World Journal
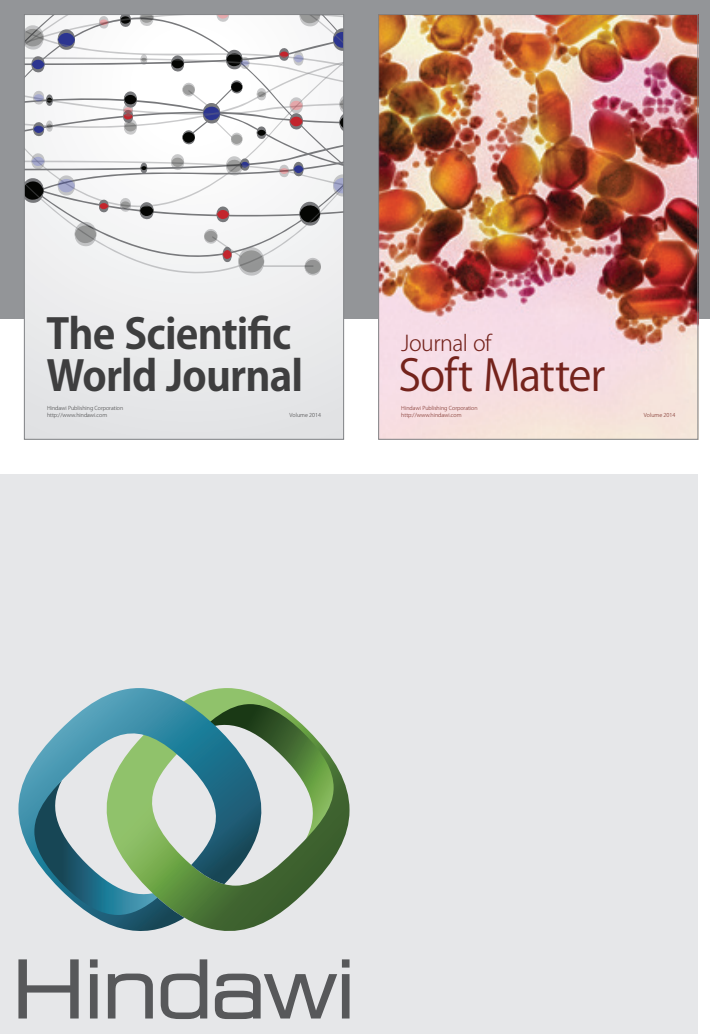

Submit your manuscripts at

http://www.hindawi.com
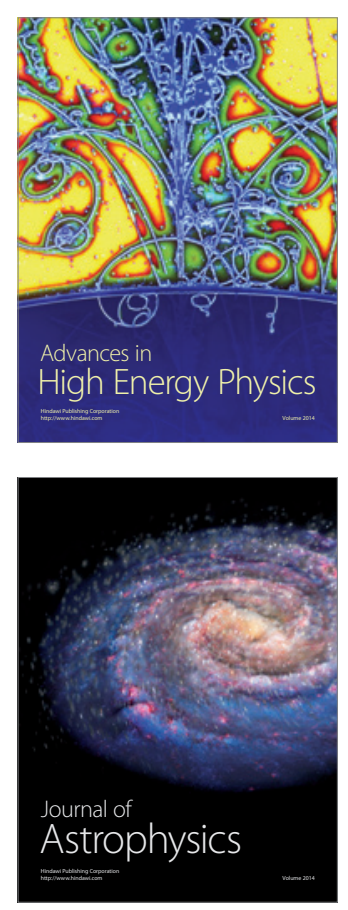
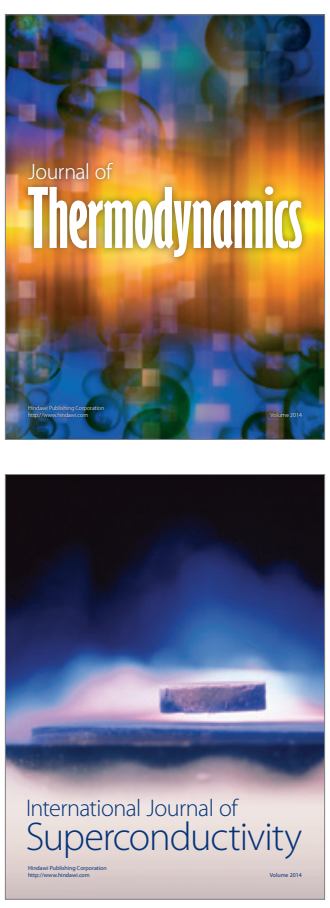
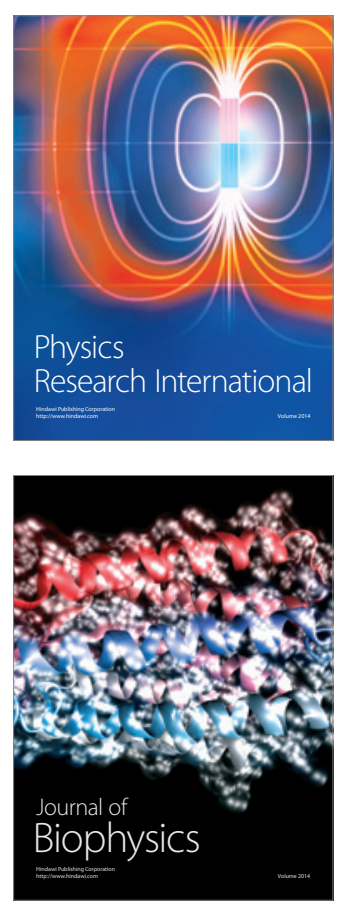
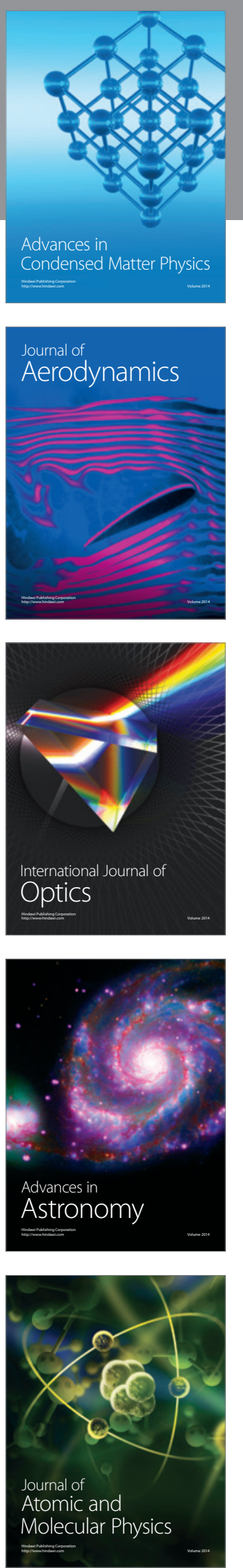